\title{
Spin Gauge Interactions as a Topological Mechanism of Super- conductivity
}

\author{
Ishita Dutta Choudhury, a \\ ${ }^{1}$ S N Bose National Centre for Basic Sciences, Block- JD, Sector-III, Salt Lake, Kolkata-700106, India
}

\begin{abstract}
We talk about a low energy, effective, topological theory of superconductivity in which a topological mass term is radiatively induced in one loop effective action. In this field theoretic model, an antisymmetric tensor field couples with the vorticity current of charged Dirac fermions in the Lagrangian. The fermion loop generates a coupling between the gauge field and the antisymmetric tensor field below an ultraviolet cut-off. The spin interactions mediated by the antisymmetric tensor field induces a mass for the photon field indicating Meissner effect. The dual antisymmetric tensor field produces a current which satisfies the relativistic version of the London equations of superconductivity. In the non-relativistic limit, the static effective potential shows a linear, always attractive term between two electrons. Thus, the theory can be considered as an alternative, low energy, effective field theory of superconductivity without spontaneous symmetry breaking.
\end{abstract}

\section{Introduction}

The discovery of fractional Quantum Hall liquids (FQHL) led us to the developement of a new sector in the theory of solid state physics[1-4]. The usual superconductivity mechanism has a local, spin zero order parameter and the theory involves spontaneous symmetry breaking. On the contrary, description of the mechanism of incompressible FQHL demands the presence of a new kind of order parameter $[5,6]$ related to the topology of the system and the mechanism cannot be explained by ordinary theory of spontaneous symmetry breaking[7].The standard theory of superconductivity was due to Bardeen, Cooper and Schrieffer. According to BCS theory, Meissner effect can be explained by the bosonization of the electrons in the form of Cooper pairs[8-10]. The interaction between the electrons and the phonons effectively generates a mutual attraction between two electrons in a limited shell in the momentum space near the Fermi surface. When this mutual attraction dominates over Coulomb repulsion, bound pairs form. The theory has a local, spin zero order parameter and the system goes through spontaneous symmetry breaking. However, in topological superconductors, the system may not have any local order parameter and there should be an associated topological field theory without involving spontaneous symmetry breaking $[11,12]$.

In $(3+1)$-dimensions, the topological theory of superconductivity involves a derivative coupling between the photon field and an antisymmetric tensor field $B_{\mu v}[13,14]$. The coefficient of this interaction plays the role of a topological mass of the photon field. The field equations of the antisymmetric tensor represent the relativistic London equations. The mechanism is similar to the famous

ae-mail: ishitadutta.choudhury@bose.res.in,iduttachoudhury2@gmail.com 
Chern-Simons theory in $(2+1)$-dimensions[15-17]. However, the Chern-Simons term breaks $P$ and $T$ invariance while BF term preserves these symmetries.

In $(2+1)$-dimensions, the radiative induction of Chern-Simons term made it possible to connect the theory with the physical systems[18, 19]. Likewise, in $(3+1)$-dimensions also, it is important to know the actual form of the interaction which effectively leads to the topological mass generation of the photon[20, 21]. In this talk, we deal with a long range interaction between the fermions which is mediated by the antisymmetric tensor field. This non-local interaction effectively generates topological BF term in the one loop effective action. This is a low energy theory and therefore is valid for the energy scales well below an ultraviolet cut-off. In the non-relativistic limit, the static effective potential has a linear attractive term between the interacting fermions[22].

\section{Spin Current}

The effective induction of topological term in the Lagrangian requires a fermion coupling with the antisymmetric tensor field $B_{\mu \nu}$. In other words, an interaction between the fermions via the antisymmetric tensor field $B_{\mu \nu}$ effectively produces topological mass for the photon. We choose the interaction as

$$
\mathcal{L}_{\text {int }}^{B}=g B_{\mu \nu} J^{\mu v}
$$

where

$$
J^{\mu \nu}=m \epsilon^{\alpha \mu \nu \sigma} \frac{\partial_{\alpha}}{\square}\left(\bar{\psi} \gamma_{\sigma} \psi\right),
$$

with $m$, the mass of the fermion. Here, the coupling of $B_{\mu v}$ with the topologically conserved current $J^{\mu v}$ has some other properties which act in favour of choosing it to use in our theory. The coupling $B_{\mu \nu} J^{\mu \nu}$, along with $P$ and $T$ symmetry has an additional vector gauge invariance under the transformation

$$
B_{\mu \nu} \rightarrow B_{\mu v}+\partial_{\mu} \kappa_{v}-\partial_{\nu} \kappa_{\mu} .
$$

Moreover, in non-relativistic limit, the spin contribution to the $0 i$-th components $J^{0 i}$ are the spin magnetic moment densities of the fermions if the fermions are localised.

Thus, a non-local spin interaction between the fermions, mediated by antisymmetric tensor field can be used to generate a topological photon mass.

\section{Calculations}

The original form of the complete Lagrangian density used to construct the fully relativistic model is given by

$$
\mathcal{L}=\bar{\psi} \gamma^{\mu}\left(i \partial_{\mu}+e A_{\mu}\right) \psi-m \bar{\psi} \psi+g B_{\mu \nu} J^{\mu \nu}-\frac{1}{4} F_{\mu \nu} F^{\mu \nu}+\frac{1}{12} H_{\mu \nu \alpha} H^{\mu \nu \alpha}
$$

where $H_{\mu v \alpha} \equiv \partial_{[\mu} B_{v \alpha]}$ is the Kalb-Ramond [24] gauge invariant field strength for the antisymmetric tensor and $g$ is the dimensionless coupling constant for the spin interactions. As in [21], this model is a low-energy effective theory, valid for energy scales below some ultraviolet cutoff $\Lambda$. By partial integration, the antisymmetric tensor interaction can be written as

$$
\mathcal{L}_{\text {int }}^{B}=g B_{\mu \nu} J^{\mu \nu}=\frac{2 m g}{\square} F_{\mu} J^{\mu},
$$


where $F_{\mu}=(1 / 2) \epsilon_{\mu \nu \alpha \beta} \partial^{v} B^{\alpha \beta}$ is the dual Kalb-Ramond field strength. One can combine the two terms for the interaction of the fermions, one with the dual Kalb -Ramond field strength and the other with ordinary gauge field, to write the total interaction term of the fermions in terms of an effective photon field $A_{\text {eff }}^{\mu}$ which is a combination of the ordinary photon field and the inverse d'Alembertian of the dual Kalb-Ramond field strength.

Integrating out the fermions, the one-loop induced action can be obtained as

$$
\Gamma^{1-\text { loop }}=\frac{e^{2}}{4} \frac{1}{12 \pi^{2}} \ln \frac{\Lambda^{2}}{m^{2}} \int d^{4} x\left(F_{\text {eff }}\right)_{\mu v}\left(F_{\text {eff }}\right)^{\mu v},
$$

where $F_{\text {eff }}^{\mu v}$ is the field strength of the effective photon field.

Expansion of the one-loop induced action in terms of the original gauge fields present in the action produces three terms. Combining those three terms with the original action the 1-loop effective Lagrangian can be written as

$$
\mathcal{L}=-\frac{1}{4 e_{\mathrm{ph}}^{2}} F_{\mu \nu} F^{\mu \nu}+\frac{g_{\mathrm{ph}} m}{2 \pi} B_{\mu \nu} \epsilon^{\mu \nu \rho \sigma} \partial_{\rho} A_{\sigma}+\frac{1}{12} H_{\mu \nu \alpha} H^{\mu \nu \alpha}+\frac{6 g_{\mathrm{ph}}^{2} m^{2}}{\ln \frac{\Lambda^{2}}{m^{2}}} F_{\mu} \frac{1}{\square} F^{\mu}
$$

where

$$
e_{\mathrm{ph}}^{2}=e^{2}\left(1+\frac{e^{2}}{12 \pi^{2}} \ln \frac{\Lambda^{2}}{m^{2}}\right)
$$

is the QED renormalized charge and

$$
g_{\mathrm{ph}}=\frac{g}{6 \pi} \ln \frac{\Lambda^{2}}{m^{2}} .
$$

Here, the second term in Eq. (7) can be identified as the topological BF term. The induced nonlocal term is represented as a Gaussian integral over a new massless vector gauge field. Using a proper set of combinations of the auxilliary gauge field and the photon field the Lagrangian is finally diagonalized.

Thus, the resulting one-loop Lagrangian decouples into two parts,

$$
\begin{aligned}
\mathcal{L} & =\mathcal{L}_{1}+\mathcal{L}_{2}, \\
\mathcal{L}_{1} & =-\frac{1}{4 e_{1}^{2}} F_{\mu \nu} F^{\mu \nu}+\frac{g_{\mathrm{ph}} m}{2 \pi} B_{\mu \nu} \epsilon^{\mu \nu \rho \sigma} \partial_{\rho} A_{\sigma}+\frac{1}{12} H_{\mu \nu \alpha} H^{\mu \nu \alpha}, \\
\mathcal{L}_{2} & =-\frac{1}{4 e_{2}^{2}} G_{\mu \nu} G^{\mu \nu}
\end{aligned}
$$

where

$$
\begin{aligned}
& e_{1}^{2}=e_{\mathrm{ph}}^{2} \frac{1+\frac{4 e_{\mathrm{ph}}^{2}}{12 \pi^{2}} \ln \frac{\Lambda^{2}}{m^{2}}}{\frac{4 e_{\mathrm{ph}}^{2}}{12 \pi^{2}} \ln \frac{\Lambda^{2}}{m^{2}}}, \\
& e_{2}^{2}=e_{\mathrm{ph}}^{2}\left(1+\frac{4 e_{\mathrm{ph}}^{2}}{12 \pi^{2}} \ln \frac{\Lambda^{2}}{m^{2}}\right) .
\end{aligned}
$$


Eq. (10) corresponds to the topological mass generation model with an effective charge $e_{1}$ that reduces to the usual renormalized electron charge, $e_{1} \rightarrow e_{\mathrm{ph}}$, for $m \ll \Lambda$. The second part written in Eq. (11)has a free, massless gauge field with field strength $G_{\mu \nu}$.

The equations of motion from the Eq. (10) can be written as

$$
\begin{aligned}
\partial_{\mu} F^{\mu v} & =-\frac{e_{1}^{2} g_{\mathrm{ph}} m}{6 \pi} \epsilon^{v \mu \alpha \beta} H_{\mu \alpha \beta}, \\
\partial_{\mu} H^{\mu \alpha \beta} & =\frac{g_{\mathrm{ph}} m}{2 \pi} \epsilon^{\alpha \beta \mu \nu} F_{\mu \nu},
\end{aligned}
$$

from which one can derive $[7,13,14,25]$ :

$$
\left[\square+\left(\frac{e_{1} g_{\mathrm{ph}} m}{\pi}\right)^{2}\right] F_{\mu \nu}=0 .
$$

Eq.(15) shows that the field strength $F_{\mu \nu}$ for the photon satisfies the Klein-Gordon equation with mass given by $\frac{e_{1} g_{\mathrm{ph}} M}{\pi}$. By a similar analysis we find that $H^{\mu v \alpha}$ also satisfies the equation of motion with the same mass.

From eq.(13) we also recognize that the dual of the Kalb-Ramond field strength acts as charged current for the photon field:

$$
\begin{aligned}
\partial_{\mu} F^{\mu v} & =J^{v}, \\
J^{v} & \equiv \frac{-e_{1}^{2} g_{\mathrm{ph}} m}{\pi} H^{v}, \\
& =\frac{-e_{1}^{2} g_{\mathrm{ph}} m}{6 \pi} \epsilon^{\nu \mu \alpha \beta} H_{\mu \alpha \beta} .
\end{aligned}
$$

Substituing eq.(16) in the equation eq.(14) we obtain:

$$
\begin{aligned}
\epsilon^{\mu \nu \alpha \beta} \partial_{\alpha} J_{\beta} & =-\left(\frac{e_{1} g_{\mathrm{ph}} m}{\pi}\right)^{2} \tilde{F}_{\mu \nu}, \\
\tilde{F}_{\mu \nu} & =\frac{1}{2} \epsilon^{\mu \nu \alpha \beta} F_{\alpha \beta}
\end{aligned}
$$

which is nothing but a relativistic version of the London equations for superconductivity.

\section{Static effective potential}

The interaction between the fermions mediated by the antisymmetric tensor field $B_{\mu \nu}$ in our low energy, effective theory for Meissner effect, has a non-local nature. This non-local nature of the current provokes an interesting question regarding the semi-classical picture of the theory, because of another well-known fact about the antisymmetric tensor field. Just as the ordinary gauge potential couples to worldlines of charged particles, the antisymmetric tensor field $B_{\mu \nu}$ couples with worldsheets which are traced out by one dimensional strings. Actually, the antisymmetric tensor field $B_{\mu \nu}$ was first postulated to describe interstring interactions. Our fermion coupling with the $B$ field is not localized at a point and therefore, the question arises such as whether the system contains string-like objects and what would the strings be made of.

For calculating the static effective potential between the interacting fermions, we integrate out the gauge fields to obtain the effective action as 


$$
S[\psi, \bar{\psi}]=S_{D}[\psi, m]+S_{A}[\psi, \bar{\psi}]+S_{B}[\psi, \bar{\psi}],
$$

where $S_{D}[\psi, m]$ corresponds to the kinetic term and the mass term for the fermion, and

$$
\begin{aligned}
& S_{A}[\psi, \bar{\psi}]=\frac{1}{2} \int \frac{d^{4} k}{(2 \pi)^{4}} J^{\sigma}(-k) \frac{e^{2}}{k^{2}} J_{\sigma}(k), \\
& S_{B}[\psi, \bar{\psi}]=\int \frac{d^{4} k}{(2 \pi)^{4}} J^{\sigma}(-k) \frac{g^{2} m^{2}}{k^{4}} J_{\sigma}(k) .
\end{aligned}
$$

In the non-relativistic limit, we expand the fermion fields in terms of three dimensional creation amd annihilation operators and note that the leading contribution to the effective potential is from the $J^{0}$ component. The reason is that the components $J^{i}$ are made of the lower components of the Dirac spinor and therefore can be neglected in the non-relativistic limit as their energies are much lower than their mass. In three dimensional momentum space, the resulting static potential has the form

$$
V(\mathbf{k})=\frac{e^{2}}{|\mathbf{k}|^{2}}-\frac{g^{2} m^{2}}{|\mathbf{k}|^{4}},
$$

which leads to a linear term in the expression for the static potential in three dimensional coordinate space as

$$
V(r)=\frac{e^{2}}{4 \pi r}+\frac{g^{2} m^{2} r}{4 \pi} .
$$

The sign of the linear potential remains same for interactions between fermions with like charges and for those with opposite charges. A linear potential is commonly associated with string-like objects.

\section{Conclusion}

We have found that a long range interaction between fermions, mediated by an antisymmetric tensor field effectively induces a derivative coupling between the photon field and the antisymmetric tensor field. The equations of motion of the photon field can be used to write the Klein- Gordon equation as a massive wave equation which explicitly shows the presence of a topological mass of the photon field. The dual Kalb-Ramond field strength acts as a current to the photon field and that current satisfies the relativistic version of the London equations of superconductivity. In the non-relativistic limit, the long range interaction mediated by the antisymmetric tensor field gives rise to linear term in the static effective potential between two electrons. This linear term is independent of the spin orientations of the interacting electrons and is always attractive in nature irrespective of the electric charges of the interaction particles. Whenever this attractive force dominates over Coulomb repulsion, bound paires may be found. Thus, the theory can be considered as an alternative, low energy, effective field theory for the superconductivity which does not involve spontaneous symmetry breaking.

\section{References}

[1] S. M. Girvin, A. H. MacDonald, Phys. Rev. Lett. 58, 1252 (1987).

[2] S. C. Zhang, T. H. Hansson, S. Kivelson, Phys. Rev. Lett. 62, 82 (1989). 
[3] S. C. Zhang, Int. J. Mod. Phys. B 06, 25 (1992).

[4] X. L. Qi, E. Witten and S. C. Zhang, Phys. Rev. B 87, 134519 (2013).

[5] X. G. Wen, Advances in Physics 44, 405 (1995).

[6] X.-G. Wen, Int. Jour. Mod. Phys. B 6, 1711 (1992).

[7] M. C. Diamantini, P. Sodano and C. A. Trugenberger, Eur. Phys. J. B 53, 19 (2006).

[8] L. N. Cooper, Phys. Rev. 104, 1189 (1956).

[9] J. Bardeen, L. N. Cooper and J. R. Schrieffer, Phys. Rev. 108, 1175 (1957).

[10] N. N. Bogolyubov, Sov. Phys. JETP 7, 41 (1958) [Zh. Eksp. Teor. Fiz. 34, 58 (1958)] [Front. Phys. 6, 399 (1961)].

[11] T. H. Hansson, V. Oganesyan and S. L. Sondhi, Annals Phys. 313, 497 (2004).

[12] D. Birmingham, M. Blau, M. Rakowski and G. Thompson, Phsy. Rep. 209 129(1991).

[13] T. J. Allen, M. J. Bowick and A. Lahiri, Mod. Phys. Lett. A 6, 559 (1991).

[14] M. Bergeron, G. W. Semenoff and R. J. Szabo, Nucl. Phys. B 437, 695 (1995).

[15] R. Jackiw and S. Templeton, Phys. Rev. D 23, 2291 (1981).

[16] S. Deser, R. Jackiw and S. Templeton, Phys. Rev. Lett. 48, 975 (1982).

[17] S. Deser, R. Jackiw and S. Templeton, Annals Phys. 140, 372 (1982) Erratum: [Annals Phys. 185, 406 (1988)].

[18] A. N. Redlich, Phys. Rev. Lett. 52, 18 (1984).

[19] A. N. Redlich, Phys. Rev. D 29, 2366 (1984).

[20] M. C. Diamantini, G. Guarnaccia and C. A. Trugenberger, J. Phys. A 47, 092001 (2014).

[21] I. D. Choudhury, M. C. Diamantini, G. Guarnaccia, A. Lahiri and C. A. Trugenberger, JHEP 1506, 081 (2015).

[22] Chandrasekhar Chatterjee, I. D. Choudhury and Amitabha Lahiri, [In preparation].

[23] M. Leblanc, R. MacKenzie, P. K. Panigrahi and R. Ray, Int. J. Mod. Phys. A 9, 4717 (1994).

[24] M. Kalb and P. Ramond, Phys. Rev. D 9, 2273 (1974).

[25] M. C. Diamantini, P. Sodano and C. A. Trugenberger, New J. Phys. 14, 063013 (2012). 